\title{
MANAGING THE KNEE ARTHROPLASTY EPISODE OF CARE THROUGH AN INTEGRATED KNOWLEDGE NETWORK
}

\author{
Nilmini Wickramasinghe ${ }^{\mathrm{a}, \mathrm{b}}$, Dominic King ${ }^{\mathrm{c}}$, Nicholas Piuzzic ${ }^{\mathrm{c}}$ Judith Welsh $^{\mathrm{c}}$ \\ and Jonathan Schaffer ${ }^{\mathrm{c}}$ \\ a Swinburne University of Technology, Australia \\ ${ }^{b}$ Epworth HealthCare, Australia \\ ${ }^{c}$ The Cleveland Clinic, USA
}

\begin{abstract}
The reality of today's healthcare environment includes escalating costs and increasing pressure to deliver high quality, high value, and patient-centered care while simultaneously improving access and controlling costs. As the population ages, our knees degenerate and patients often present for knee replacement, known as knee arthroplasty. The growing aging population translates into significant increases in the demand of knee arthroplasty. For prudent knee arthroplasty episode of care management, the usual mechanistic approach, focusing on a cause to effect chain of actions are insufficient to provide statistical modelling that can forecast patient clinical outcomes including complication risks. Collecting relevant data will facilitate decisions by patients and providers, yet in knee arthroplasty, there is incomplete physiologic and psychosocial data that charts the entire episode of care from presentation to full recovery. To address the episode of care for knee arthroplasty, we design and develop a sophisticated integrated knowledge network that can be used to statistically model care pathways that will in turn ensure effective, efficient and efficacious processes. Specifically, we focus on the first step of the episode of care which is surgical decision making that should be shared by patients and providers. Engagement of the patient is desired and considered critical to a successful outcome, yet few programs exist which model specific mitigatable variables such as body mass index, co-morbidities and patient participation in the postoperative rehabilitation. Our integrated knowledge network is based on integrating a well-known industrial and military decision support tool into our intelligence continuum yielding real time integration of critical data and information while simultaneously capturing tacit knowledge that can enhance optimal clinical outcomes and patient experience.
\end{abstract}

\section{KEYWORDS}

Episode of Care, Patient Operative Journey, Healthcare Value Proposition, Intelligence Continuum, Knowledge Management

\section{INTRODUCTION}

When patients experience knee pain due to degenerative changes in their joint anatomy, there are a number of acceptable treatment modalities and risk mitigation strategies that should be addressed prior to undergoing knee arthroplasty. In a value based payment model, there is an inherent bias for patient management to focus on these modalities and risk management strategies while the converse is also true for recommending knee arthroplasty. However, patient knowledge is often limited or misguided based on inconsistent information sources. The mechanistic approach entails the cause of the patient's pain - knee degeneration - and the effect - recommending addressing the pain with a joint replacement consisting of resurfacing the knee joint surfaces. There are many variables in the knee joint resurfacing alone that are left to the surgeon without shared decision making around key aspects such as cemented or uncemented implantation, resurfacing the patella or not resurfacing, perioperative expectations for pain management and rehabilitation, discharge planning and home care or outpatient rehabilitation, equipment needs at home, and comorbidity impact on healing and well-being. Each of these factors can be addressed through a statistical model, yet that has at best on partially permeated the arthroplasty community. Improving patient education through an integrated knowledge network should serve to improve both clinical outcomes and the patient experience. 
Unstructured decision making in dynamic and complex environments is not only challenging but in almost every situation the decision maker is undoubtedly faced with information inferiority (Award and Ghaziri, 2004). Germane knowledge, pertinent information and relevant data are critical while the value of harnessing knowledge and embracing the tools of knowledge management are essential to ensuring efficiency and efficacy in the decision-making process (Wickramasinghe et al., 2009). Given today's healthcare contexts, where tremendous data is generated from multiple sources, the demand for decreased processing time is increased when making critical decisions, many of which may have far reaching live altering consequences, more than ever is an integrated knowledge network a strategic necessity. Drawing upon a process centric perspective to knowledge generation and combining this with the intelligence continuum, our model offers an integrated knowledge network framing to support prudent and effective episode of care management for knee arthroplasty care delivery (Wickramasinghe and Schaffer, 2008).

\section{BACKGROUND}

Before it is possible to design and develop an appropriate integrated knowledge network that supports the episode of care, it is necessary to first understand key, and often unique, aspects of the healthcare industry, the significant challenges and the underlying healthcare value proposition. In sharp contrast to other industries, healthcare has a unique structure in which the receiver of the services (i.e. the patient) is often not the predominant payer for those services (i.e. the insurance company or government) (Wickramasinghe and Schaffer, 2010). Further, healthcare interventions are typically complex and involves a variety of players such as; providers, payers, patients and regulators (Wickramasinghe et al., 2008; 2009). This in turn leads to economic dilemmas such as moral hazard, orthogonal considerations pertaining to cost versus quality and information asymmetry which in turn have the potential to create obstacles intentionally or not when trying to deliver efficient and effective healthcare (Boyd, 1976; von Lubitz and Wickramasinghe, 2006). To address these concerns and provide efficient, effective and efficacious care delivery, relevant data, pertinent information and germane knowledge play a vital role and can only be obtained via the careful structure and design of a technology enabled system; we have defined as the intelligence continuum (Alberts et al., 2000; Cebrowski and Garstka, 1998; Boyd, 1987).

Succinctly, the intelligence continuum relies on multispectral inputs from patient data, established clinical practice guidelines, environmental data and psycho-social data, on which key analytics techniques, powered by latest technologies and tools, are applied to identify critical patterns, insights and factors to support expert judgement in deciding best practice to adopt along the clinical episode of care for the specific presenting patient (Wickramasinghe and Schaffer, 2010). In following such a precise and systematic protocol, it also becomes possible to simultaneously deliver on a healthcare value proposition of better access, quality and value of care delivery as well as ensure heightened clinical outcomes ensue and patient satisfaction is high. Integral to the successful application of the intelligence continuum, is a process-centric perspective of knowledge creation which in turn leads to an integrated knowledge network (Wickramasinghe and Schaffer, 2010).

\section{THE PROCESS-CENTRIC PERSPECTIVE OF KNOWLEDGE}

A process centric approach to knowledge creation serves to combine critical people centric and technology centric perspectives and at the same time emphasizes the dynamic and on-going nature of the process (Wickramasinghe et al., 2009). This is particularly relevant for healthcare contexts as a healthcare intervention should not be looked at just one point in time but the progression of the patient along their care recovery journey (Wickramasinghe et al 2008). This process-centric perspective is grounded in the pioneering work of Boyd and his OODA Loop; a conceptual framework that maps critical process required to support rapid decision making and extraction of essential and germane knowledge while filtering out extraneous noise (Alberts et al., 2000; Cebrowski and Garstka, 1998; Boyd, 1987; Wickramasinghe and Schaffer, 2006). Boyd developed the OODA loop with the premise that when an entity makes appropriate decisions the fastest, thereby completing the OODA loop in the shortest cycle time, they will become the victor (Alberts et al., 2000; Cebrowski and Garstka, 1998; Boyd, 1987). As in aviation, medical care involves continuous cycles of interaction between a disease or condition, the patient and the treatment team. 
The OODA Loop is based on a cycle of four interrelated stages essential to support critical analysis and rapid decision making that revolve in both time and space: Observation followed by Orientation, then by Decision, and finally Action (OODA) (Alberts et al., 2000; Cebrowski and Garstka, 1998; Boyd, 1987). At the Observation and Orientation stages, implicit and explicit inputs are gathered or extracted from the environment (Observation) and then converted into coherent information (Orientation) (Alberts et al., 2000; Cebrowski and Garstka, 1998; Boyd, 1987). The latter determines the sequential determination (knowledge generation) and Action (practical implementation of knowledge) steps (Alberts et al., 2000; Cebrowski and Garstka, 1998; Boyd, 1987, figure 1). The outcome of the Action stage then affects, in turn, the character of the starting point (Observation) of the next revolution in the forward progression of the continuous loop (Alberts et al., 2000; Cebrowski and Garstka, 1998; Boyd, 1987). Appropriate metrics are assessed and the cycle continues with a new cycle of observation (Wickramasinghe and Schaffer, 2006). In the patient context, a clinician completing the OODA loop in the shortest time will diagnose and treat the patient before situations change leading to an optimal clinical outcome (Wickramasinghe and Schaffer, 2010).

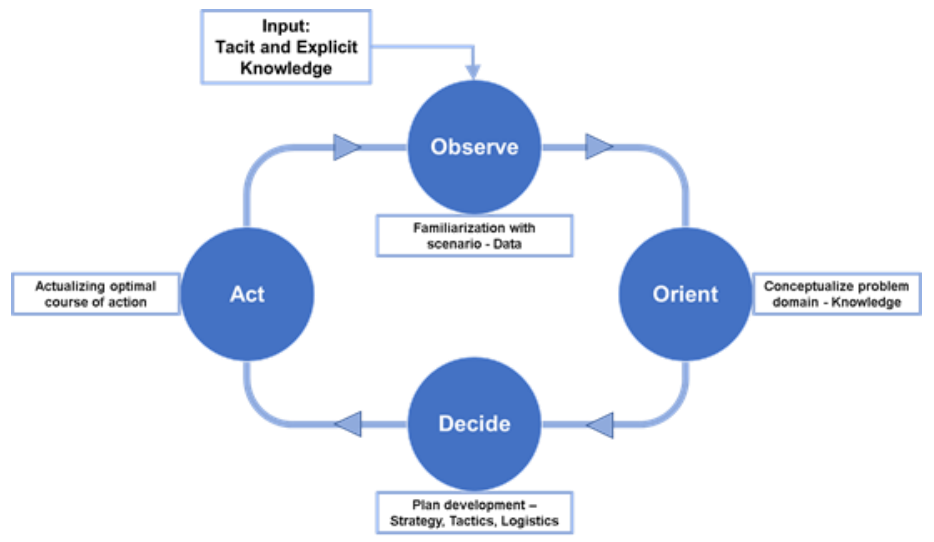

Figure 1. Process Perspective of Knowledge Generation (adapted from Wickramasinghe and Schaffer, 2006)

The focus of this paper is to establish an integrated knowledge network framing to support prudent episode of care management for knee arthroplasty care delivery. Hence, this paper serves to answer the research question: How can we develop a suitable integrated knowledge network to support orthopaedic episode of care management?

\section{METHOD}

The nature of this study is exploratory, focusing on longitudinal data gathered from an orthopaedic site which is part of a large multi-specialty group practice in the USA. Thematic analysis is used to identify key aspects of the data during the episode of care management to make up the primary phases of the integrated knowledge network. The study has received necessary ethics approvals. An Action Research Methodology (Rowell et al., 2017) combined with a design science research methodology (DSRM) (Hevner and Chatterjee, 2010) is adopted to support the development of the proposed framework. DSRM is applied to design and develop an appropriate strategy to adapt the components of the intelligence continuum to this context as well as to then develop a prototype decision support solution. Action research, is an interactive inquiry process that balances problem solving actions implemented in a collaborative context with data-driven collaborative analysis or research to understand underlying causes enabling future predictions about personal and organisational change (Crossing the Quality Chasm, 2001). The DSRM is adopted to guide the cycles of problem/opportunity determination (relevance cycle), solution design and evaluation examine (design cycle), using the knowledge base to inform design (rigor cycle) (Hevner and Chatterjee, 2010; Hevner et al., 2004).

The orthopaedic episode of care management model draws upon the application of knowledge management (KM) principles in combination with Boyd's OODA Loop (Observe, Orient, Decide, Act). This model creates an Intelligence Continuum (Wickramasinghe and Schaffer, 2006) which serves to provide an integrated, systematic and dynamic model for ensuring that the healthcare decision maker is always provided with 
appropriate and necessary knowledge elements such that healthcare decision making processes can lead to optimal outcomes (figure 1). In healthcare, an added complication is that it is typical not to have all the data, it is what is available at the time that must be used to make a decision. This makes the tacit knowledge of the surgeon critical in accounting in some manner for what data might or might not be missing or just not available when a decision needs to be made. Clearly, the more complete or the smaller the gap the more likely the optimal action will be selected.

Focusing on the initiation point for knee arthroplasty provides the impetus to launch the patient on the optimal trajectory. Should mitigatable or modifiable risk factors not be understood or addressed, the clinical outcome and patient experience are likely to be compromised or at best suboptimal. Value models should focus on this first step however have tended to focus downstream in the process flow. For example, discharge planning should begin when surgery is being discussed and scheduled and not once surgery is completed (refer to figure 2).



Figure 2. The knee arthroplasty context of the Integrated Knowledge Network (adapted from King and Schaffer 2020)

\section{CASE EXAMPLE}

Knee arthroplasty has become a predictable operative experience resulting in predictable outcomes with excellent clinical outcomes. However, BMI, Hb, HbAlc, Albumin, ASA score, opioid dependence and smoking status are examples of factors associated with increased risks for knee arthroplasty outcomes. These preoperatively modifiable risk factors should be evaluated before surgery by identifying the scheduling of the index procedure, automatically ordering the tests or consults to optimize these risk factors and then permit surgical scheduling once optimization is achieved or an acceptable override is documented. "Surgical optimization", the concept of leveraging outcomes research, quality improvement research and safety and risk mitigation research to outline the value of recognizing and managing modifiable risk factors prior to surgical intervention, is enabled by the proffered integrated knowledge network so that "un-optimized" patients to be diverted to non-surgical providers for modifiable risk factor management. Previously, we have modelled the journey from diagnosis through pre, peri and post-surgery and rehab and then to home. The micro level specific patient data is captured at the time of service, leading to automated recommendations. The meso level tracks and incorporates the patient response to the recommendations. At the macro level, data capture facilitates comparing these preoperative risk factors to postoperative outcomes providing further input for the statistical model. Through machine learning, descriptive, predictive and prescriptive analytics are provided to further refine the episode of care. These data also enable benchmarking to national registries to determine performance, outcomes, quality and patient experience. 


\section{DISCUSSION AND CONCLUSIONS}

To improve the efficiency, effectiveness and efficacy of patient care for those patients requiring knee replacement, every healthcare process on the pathway from evaluation to operation to recovery should be optimized. Specifically, the inputs, transformation and outputs should be measured against specification for process time, scheduling, expenses, personnel, etc. need to be identified and mapped. Each individual in this processes has critical knowledge and expertise and thus it is essential this knowledge is captured as discussed above by adopting a process centric perspective to knowledge generation. From this only is it possible to make use of the opportunity to improve the knowledge spiral and use the Intelligence Continuum.

It is unsurprising that there are inherent limitations of organizational structure which must be overcome to make these improvements. The generation, representation, storage, transfer and transformation of knowledge are key steps in making the desired improvements in clinical and management practices which require multispectral data and incorporation of various techniques and technologies as outlined by the intelligence continuum. Moreover, such an approach lends itself to continuous innovation. Typically, however, the daily volume of data that is generated and accumulated is often lost, further increasing the gaps between data collection, comprehension and analysis. Boyd's OODA loop model of observation, orientation, decision, and action enables a systematic organization of the inputs and provides a robust structure for improvement and real time decision support. With an aging population coupled with longer life expectancy, it is clear that more patients with degenerative knee arthritis will need joint replacement. Given the current challenges facing healthcare delivery, surgeons and hospitals with successful clinical outcomes will use process engineering tools to identify critical path processes. Our integrated knowledge network is designed to support episodic care management and serves to address this issue and thus answers to posed research questions. Our future work will serve to test this framework in a clinical trial context to demonstrate its potential to support prudent decision making, better clinical outcomes, high patient satisfaction and consistent attainment of a healthcare value proposition around access, quality and value.

\section{REFERENCES}

Alberts DS, Garstka JJ, and Stein FP, (2000), Network Centric Wardare: Developing and Leveraging Information Superiority, CCRP Publication Series (Dept. of Defense), Washington, DC, pp 1-284 (available at http://www.dodccrp. org/publications/pdf/Alberts_NCW.pdf

Award, E. and Ghaziri, H. (2004) Knowledge Management Prentice Hall, Upper Saddle River

Boyd JR, (1976), Destruction and Creation, in R Coram "Boyd” Little, Brown \& Co, New York

Boyd JR, COL USAF, 1987. in Patterns of Conflict, unpubl Briefing (accessible as "Essence of Winning and Losing", http://www.d-n-i.net )

Cebrowski AK, Garstka JJ. (1998) Network-centric warfare: its origin and future. US Nav. Inst. Proc.1, 28-35. Boyd JR. (1987). In Patterns of Conflict, unpubl Briefing (accessible: "Essence of Winning and Losing", http://www.d-n-i.net ).

Crossing the Quality Chasm: A New Health System for the 21st Century (2001) The National Academies Press.

Hevner, A. and Chatterjee, S. (2010) Design Research in Information Systems, Springer Science+Business Media.

Hevner, A., March, S., Park, J. and Ram, S. (2004) 'Design Science in information systems research', MIS Quarterly, Vol. 28, No. 1, pp.75-105.

Rowell, L., Bruce, C., Shosh, J. M., \& Riel, M. (2017). The Palgrave international handbook of action research. New York, NY: Palgrave Macmillan.

von Lubitz D, Wickramasinghe N. (2006) Healthcare and technology: the doctrine of network-centric healthcare. Intl. J. Electronic Healthcare. 4:322-344.

Wickramasinghe N, Schaffer J. (2006) Creating Knowledge Driven Healthcare Processes with the Intelligence Continuum. Intl. J. Electronic Healthcare. 2(2):164-174.

Wickramasinghe, N. and Schaffer, J. (2010) Realizing Value Driven Patietn Centric Healthcare Delivery Through Technology, IBM Center for the Business of Government [online] http://www.businessofgovernment.org/report/realizing-value-driven-e-health-solutions (accessed February 2020).

Wickramasinghe, N., Bali, R., Lehany,B. and Gibbons, C., Schaffer, J. (2009) "HCKM Primer”, Routledge, New Jersey.

Wickramasinghe, N., J. Schaffer and R. Bali (2008) "The Health Care Intelligence continuum: key model for enabling KM initiatives and realising the full potential of SMT in healthcare delivery" IJBET vol 1 no4 pp 415-427

Wickramasinghe, N., T. Goodfellow, R. Bali, and R. Naguib (2008) From Data to Decisions: A Knowledge Management Approach for Productivity Increase in a Radiology Department” Intl. J. Biomedical Engineering and Technology Vol.1, No.3, pp.259-272 